\title{
SGLT2 Inhibitors: The Dawn of a New Era in Cardio-Metabolic Therapeutics
}

\author{
Prakash Deedwania $^{1,2}$ (D) \\ Accepted: 2 August 2021 / Published online: 30 August 2021 \\ (c) The Author(s), under exclusive licence to Springer Nature Switzerland AG 2021
}

Sodium-glucose co-transporter 2 inhibitors (SGLT2i) were developed as oral hypoglycemic agents to control hyperglycemia in patients with type 2 diabetes mellitus (T2DM), but the results of several large randomized controlled trials over the past decade have revealed that they do much more than reduce hyperglycemia and have substantial favorable cardiometabolic effects [1-12]. In this issue, Qiu et al. [13] report on a meta-analysis of ten cardiovascular outcomes trials (CVOTs) with five SGLT2i to highlight their broad actions in controlling and managing multiple cardio-metabolic perturbations. They showed that treatment with SGLT2i was associated with substantial benefits in multiple cardiometabolic and renal endpoints [13]. The authors provided detailed analyses in the paper but did not discuss the mechanistic aspects of the results observed with SGLT2i or provide information about the reasons for differences observed in their included trials. Accordingly, it will be advantageous to briefly review the mechanisms of action of SGLT2i and the findings of the major CVOTs to fully understand and put into perspective the findings described by Qiu et al. [13].

\section{Sodium-Glucose Co-Transporter 2 Inhibitors (SGLT2i), a Novel Drug Class}

SGLT2i work by blocking SGLT2, which is a low-affinity, high-capacity sodium-glucose co-transporter in the proximal renal tubules and is responsible for $90 \%$ of renal glucose reabsorption [1]. Patients with T2DM have a genetic upregulation of SGLT2 receptors, resulting in an increased renal threshold for glucose reabsorption and reduced urinary glucose excretion, contributing to hyperglycemia. SGLT2i

Prakash Deedwania

deed@ucsf.edu

1 UCSF School of Medicine, San Francisco, CA, USA

2 UCSF Fresno, Academic Suite, East Kashian Lane, Fresno, CA 93701, USA block this receptor, thereby preventing glucose reabsorption into the bloodstream and facilitating elimination of glucose in the urine (glycosuria). Because of glycosuria, these agents also have a diuretic effect and promote weight loss and blood pressure reduction due to sodium and water loss. These effects produce beneficial hemodynamic actions and are likely responsible for some of the favorable outcomes.

However, the precise mechanisms by which SGLT2i exert their beneficial effects on cardiovascular and renal outcomes remain to be established. The mechanisms behind the beneficial effects associated with SGLT2i are complex and probably multifactorial. It is evident that the benefits produced by SGLT2i clearly exceed that which would be expected by glucose lowering alone, suggesting additional mechanism(s) of action. There are multiple possibilities, and these may include improved cardiac energy metabolism, decreased inflammation, erythropoiesis, inhibition of the sympathetic nervous system, diuresis/natriuresis, blood pressure reduction, reduced arterial stiffness, afferent renal vasoconstriction, and improved kidney function, among many other potential beneficial pathways (Table 1) [14, 15, 23]. A particularly interesting potential mechanism of SGLT2i is their ability to produce a cellular response that mimics starvation [16]. The hypothesis is that SGLT2i induce a fasting pathway through activation of the low-energy sensor sirtuin 1 (SIRT1) and adenosine monophosphate-activated protein kinase (AMPK) that, in turn, reduces oxidative stress, suppresses inflammation, minimizes coronary microvascular injury, and decreases the risk of cardiomyopathy [17]. The SIRT1/AMPK pathway may also underlie the renal benefits of SGLT2i, decreasing glomerular and tubular injury and therefore decreasing the risk of nephropathy [16]. Importantly, this pathway has also been associated with stimulation of autophagy, which is a critical process to remove dangerous cellular components that can lead to organ damage [16]. The SIRT1/AMPK pathway may "trick" cells into believing that they are fasting and signal that they are hypoxic. This can potentially explain why SGLT2i appear 
Table 1 Potential mechanisms of cardio-renal benefit with sodium-glucose co-transporter-2 inhibitors [14, 15, 23]

Glycosuria with natriuresis (osmotic diuresis)

Improved cardiac loading conditions through decreased preload (natriuresis) and afterload (reduced BP)

Decreased intracellular fluid by selective removal of fluid from interstitial space with electrolyte-free water clearance

Decreased body mass and leptin production

Improved endothelial function

Improved cardiac energy metabolism

Decreased inflammation

Erythropoiesis

Inhibition of the sympathetic nervous system

Prevention of RAAS activation

Starvation mimicry through SIRT1/AMPK pathway

Direct effect on cardiac ion channels to maintain $\mathrm{Ca}+$ homeostasis in cardiac myocytes

Decreased cardiac fibrosis

Increased glomerular afferent arteriolar vasoconstriction

$A M P K$ adenosine monophosphate-activated protein kinase, $B P$ blood pressure, $C A$ calcium, $R A A S$ renin-angiotensin aldosterone system, SIRT1 sirtuin 1

to result in erythrocytosis. Additionally, in T2DM, oxygen consumption in the proximal tubule is increased because of the energy consumption associated with excessive glucose resorption, which can result in relative tubulointerstitial hypoxia. Tubulointerstitial hypoxia can in turn result in proximal tubular interstitium fibroblast dysfunction, leading to reduced erythropoietin production. SGLT2 inhibition decreases the workload of the proximal tubules and alleviates tubulointerstitial hypoxia, resulting in improved erythropoietin production by fibroblasts. Erythrocytosis has been shown in mediation analyses to be one of the most potent predictors of beneficial effects of SGLT2i in heart failure (HF) [16].

Another promising hypothesis is that SGLT2i induce sympathetic nervous system inhibition, which in turn may lead to their cardiovascular and renal benefits. In experiments with a hypertensive mouse model, SGLT2 inhibition led to a reduction in sympathetic nervous system activity, which in turn led to reduced blood pressure, decreased endothelial dysfunction, and reduced inflammation in the heart and kidney [18]. Whether this mechanism is operative in humans has yet to be determined. SLGT2i also appear to directly inhibit the sodium-hydrogen exchanger (NHE)-1 isoform in the myocardium, which may improve cardiac contractility [19]. Additionally, SGLT2i downregulate the activity of NHE3 in the kidneys and promote natriuresis, which-along with glycosuria-results in osmotic diuresis and the resultant reduction in blood pressure and weight loss.

\section{Clinical Trial Findings and Real-Life Experience with SGLT2i}

As described by Qiu et al. [13], a number of CVOTs have evaluated the effects of SGLT2i on glycemic control and their safety in patients with preexisting cardiovascular disease (CVD) and in those without CVD (but at future high risk of CVD). Qiu et al. [13] summarize the baseline characteristics and other important features of these trials in Table 2 in the electronic supplementary material associated with their article. It is important to recognize that most of the earlier CVOTs with SGLT2i were primarily conducted, as required by the US FDA mandate, to show their safety by demonstrating noninferiority for cardiovascular endpoints [2]. However, the results of the trials with SGLT2i were surprising as they showed cardio-protective effects, especially reductions in HF hospitalization and cardiovascular deaths [2-12]. Qiu et al. [13] provide the results of these trials on various outcomes of interest in Figs. 1, 2, 3, 4, 5, $6,7,8$ in their article. These results show favorable impacts from SGLT2 $i$ in various cardiovascular and renal outcomes. However, there are significant differences in several outcomes amongst some of the trials that deserve attention and explanation. For example, Fig. 1 [13] shows that the DECLARE-TIMI 58 and VERTIS CV trials did not show significant reductions in major adverse cardiovascular events compared with other trials. Although not discussed by Qiu et al. [13], this difference was most likely related to significant differences in patients' baseline characteristics, in that the patients recruited for these two trials were less sick than those recruited for the other trials. However, the findings of the network meta-analyses showed that, when the data 
from all studies were combined, efficacy was consistent and without significant heterogeneity. This is nicely highlighted in Figs. 1, 2, 3, 4, 5 [13]. Despite the differences in other cardiovascular outcomes, Fig. 6 [13] shows that, regardless of differences in baseline characteristics in various trials, there were consistent and universal reductions in hospitalization for HF, showing a dramatic reduction of approximately $30 \%$ in all studies. This is a phenomenal finding, suggesting that treatment with SGLT2i was highly and consistently effective in preventing hospitalization for HF. This kind of consistent efficacy has been demonstrated for mortality benefit in HF with only one other class of drugs: $\beta$-blockers. Furthermore, it is important to emphasize that, although not described in the paper by Qiu et al. [13], the efficacy of SGLT2i was complementary to treatment with renin-angiotensin aldosterone system (RAAS) blockers. Similarly consistent efficacy is shown (Fig. 7) [13] for the prevention of renal dysfunction. All of the effects on various events of interest are further summarized in Fig. 8 [13], which shows surface under the cumulative ranking curve for various SGLT2i.

\section{Real-Life Experience with SGLT2i in CVD-REAL and CVD-REAL 2 Studies}

The beneficial effects of SGLT2i observed in various CVOTs were also shown in the observational studies conducted in various real-life clinical settings in the multinational CVD-REAL studies [20, 21]. CVD-REAL and CVD-REAL 2 were large multinational registries that collected information on individuals with T2DM who were treated with SGLT2i. The CVD-REAL registry collected data from the USA, Norway, Denmark, Sweden, Germany, and the UK, and it showed a reduced risk of hospitalization for HF and death with SGLT2i compared with other hypoglycemic drugs in a propensity scorematched analysis [20]. The CVD-REAL 2 registry, which evaluated patients from South Korea, Japan, Singapore, Israel, Australia, and Canada, demonstrated a lower risk of death, myocardial infarction, and stroke in addition to the reduced rate of hospitalizations for HF with the use of SGLT2i [20]. These results demonstrated that the cardiovascular benefits of SGLT2i do indeed extend beyond what has been shown in the clinical trial populations and appear to be present in real-life clinical scenarios.

\section{Clinical Implications and Future Direction}

It should be noted that the scope of the benefits of SGLT2 $\mathrm{i}$ is expanding to include populations without T2DM, both in those with HF with reduced ejection fraction and in those with chronic kidney disease (CKD) [8, 22].
The addition of SGLT2i in cardio-metabolic therapeutics is nothing short of a new era, not only as the new class of antihyperglycemic agents in T2DM but also in CVD, HF, and CKD. Their consistent efficacy in HF has filled a longstanding void by providing the much-needed newer drug therapy beyond the neuro-hormonal antagonists (RAAS blockers and $\beta$-blockers) that have been the standard treatment for HF for several decades. Because SGLT2i work by different mechanism(s), their use should be complementary to these established therapies in HF. Additionally, the nephron-protective effects of SGLT2i would also be beneficial in most patients with CVD and HF, as many of these patients have concomitant CKD and renal failure.

Based on the available body of evidence from the CVOTs with SGLT2i, most national and international guidelines now recommend their use as second-line (after metformin) therapy in the treatment of T2DM. Additionally, because of their cardio-protective and nephron-protective effects, SGLT2i are now recommended as standard therapy for most patients with $\mathrm{HF}$ as well as those at future risk of $\mathrm{HF}$ and those with CKD. The results of some recent trials showed that these beneficial effects of SGLT2i were seen regardless of the presence of underlying T2DM. The ongoing trials will further elucidate the cardiovascular and renal effects of these agents in individuals without T2DM.

\section{Declarations}

Funding No external funding was used in the preparation of this manuscript.

Conflict of interest Professor Deedwania has no potential conflicts of interest that might be relevant to the contents of this manuscript.

Ethics approval Not applicable.

Consent Not applicable.

Availability of data and material Not applicable

Author contributions Professor Deedwania conceptualized and wrote this invited editorial.

\section{References}

1. Abdul-Ghani MA, Norton L, Defronzo RA. Role of sodium-glucose cotransporter 2 (SGLT 2) inhibitors in the treatment of type 2 diabetes. Endocr Rev. 2011;32:515-31.

2. Deedwania P, Acharya T. Cardiovascular protection with antihyperglycemic agents. Am J Cardiovasc Drugs. 2019;19:249-57.

3. Zinman B, Wanner C, Lachin JM, EMPA-REG OUTCOME Investigators, et al. Empagliflozin, cardiovascular outcomes, and mortality in type 2 diabetes. N Engl J Med. 2015;373:2117-28.

4. Fitchett D, Zinman B, Wanner C, et al. Heart failure outcomes with empagliflozin in patients with type 2 diabetes at high 
cardiovascular risk: results of the EMPA-REG OUTCOME trial. Eur Heart J. 2016;37:1526-34.

5. Neal B, Perkovic V, Mahaffey KW, CANVAS Program Collaborative Group, et al. Canagliflozin and cardiovascular and renal events in type 2 diabetes. N Engl J Med. 2017;377(7):644-57.

6. Wanner C, Inzucchi SE, Lachin JM, EMPA-REG OUTCOME Investigators, et al. Empagliflozin and progression of kidney disease in type 2 diabetes. N Engl J Med. 2016;375(4):323-34.

7. Perkovic V, Jardine MJ, Neal B, et al. Canagliflozin and renal outcomes in type 2 diabetes and nephropathy. $\mathrm{N}$ Engl J Med. 2019;380:2295-306.

8. McMurray JJV, Solomon SD, Inzucchi SE, et al. Dapagliflozin in patients with heart failure and reduced ejection fraction. N Engl J Med. 2019;381:1995-2008.

9. Heerspink HJL, Stefansson BV, Correa-Rotter R, et al. Dapagliflozin in patients with chronic kidney disease. N Engl J Med. 2020;383:1436-46.

10. Packer M, Anker SD, Butler J, et al. Cardiovascular and renal outcomes with empagliflozin in heart failure. N Engl J Med. 2020;383:1413-24.

11. Bhatt DL, Szarek M, Pitt B, et al. Sotagliflozin in patients with diabetes and chronic kidney disease. N Engl J Med. 2020;384:129-39.

12. Cannon CP, Pratley R, Dagogo-Jack S, et al. Cardiovascular outcomes with ertugliflozin in type 2 diabetes. N Engl J Med. 2020;383:1425-35.

13. Qiu M, Ding LL, Zhou HR. Comparative efficacy of five SGLT2i on cardiorenal events: a network meta-analysis based on ten CVOTs. Am J Cardiovasc Drugs. 2021. https://doi.org/10.1007/ s40256-021-00484-8.

14. Lopaschuk GD, Verma S. Mechanisms of cardiovascular benefits of sodium glucose co-transporter 2 (SGLT2) Inhibitors: a stateof-the-art review. JACC Basic Transl Sci. 2020;5:632-44.

15. Cherney DZ, Odutayo A, Aronson R, et al. Sodium glucose cotransporter-2 inhibition and cardiorenal protection: JACC review topic of the week. J Am Coll Cardiol. 2019;74:2511-24.
16. Packer M. SGLT2 inhibitors produce cardiorenal benefits by promoting adaptive cellular reprogramming to induce a state of fasting mimicry: a paradigm shift in understanding their mechanism of action. Diabetes Care. 2020;43:508-11.

17. Zhou H, Wang S, Zhu P, et al. Empagliflozin rescues diabetic myocardial microvascular injury via AMPK-mediated inhibition of mitochondrial fission. Redox Biol. 2018;15:335-46.

18. Herat LY, Magno AL, Rudnicka C, et al. SGLT2 inhibitor-induced sympathoinhibition: a novel mechanism for cardiorenal protection. JACC Basic Transl Sci. 2020;5:169-79.

19. Sharma A, Verma S. Mechanisms by which glucagon-likepeptide-1 receptor agonists and sodium-glucose cotransporter-2 inhibitors reduce cardiovascular risk in adults with type 2 diabetes mellitus. Can J Diabetes. 2020;44:93-102.

20. Kosiborod M, Cavender MA, Fu AZ, CVD-REAL Investigators and Study Group, et al. Lower risk of heart failure and death in patients initiated on sodium-glucose cotransporter- 2 inhibitors versus other glucose-lowering drugs: the CVD-REAL Study (Comparative Effectiveness of Cardiovascular Outcomes in New Users of Sodium-Glucose Cotransporter-2 Inhibitors). Circulation. 2017;136(3):249-59.

21. Kosiborod M, Lam CSP, Kohsaka S, CVD-REAL Investigators and Study Group, et al. Lower cardiovascular risk associated with SGLT-2i in >400,000 Patients: the CVD-REAL 2 Study. J Am Coll Cardiol. 2018;71:2628-39.

22. Herrington WG, Preiss D, Haynes R, et al. The potential for improving cardio-renal outcomes by sodium-glucose cotransporter-2 inhibition in people with chronic kidney disease: a rationale for the EMPA-KIDNEY study. Clin Kidney J. 2018;11:749-61.

23. Ojha U, Reyes L, Eyenga F, et al. Diabetes, heart failure and beyond: elucidating the cardioprotective mechanisms of sodium glucose cotransporter 2 (SGLT2) inhibitors. Am J Cardiovasc Drugs. 2021. https://doi.org/10.1007/s40256-021-00486-6. 